\title{
Diagnostic accuracy, safety and utilisation of respiratory physician-delivered thoracic ultrasound
}

\author{
Najib M Rahman, ${ }^{1}$ Aran Singanayagam, ${ }^{1}$ Helen E Davies, ${ }^{1}$ John M Wrightson, ${ }^{1}$ \\ Eleanor K Mishra, ${ }^{1}$ Y C Gary Lee, ${ }^{2}$ Rachel Benamore, ${ }^{3}$ Robert J 0 Davies, ${ }^{1}$ \\ Fergus $\mathrm{V}$ Gleeson ${ }^{3}$
}

\begin{abstract}
- Additional methods, figures and tables are published online only. To view these files please visit the journal online http:// thorax.bmj.com/content/vol65/ issue 5
\end{abstract}

${ }^{1}$ Oxford Centre for Respiratory Medicine and Oxford Pleural Diseases Unit, Churchill Hospital, Oxford, UK ${ }^{2}$ University of Western Australia, Department of Medicine, Australia ${ }^{3}$ Department of Radiology, Oxford Centre for Respiratory Medicine, Churchill Hospital, Oxford, UK

\section{Correspondence to} Dr Najib Rahman, Oxford Centre for Respiratory Medicine, Churchill Hospital, Oxford OX3 7LJ, UK;

naj_rahman@yahoo.co.uk

Received 4 October 2009 Accepted 22 February 2010

\begin{abstract}
Background Thoracic ultrasound-guided pleural procedures are associated with fewer adverse events than 'blind' procedures for patients with pleural effusion. Ultrasound is increasingly practised by respiratory physicians but there has been no prospective assessment of its safety and diagnostic accuracy when delivered by respiratory physicians.

Methods The activity level, safety and diagnostic accuracy of thoracic ultrasound delivered by respiratory physicians were prospectively assessed. Diagnostic accuracy was assessed using a stepwise pragmatic approach (recording if pleural fluid was obtained or effusion was present on another radiological modality). In the absence of the above, ultrasound clips were reviewed by a blinded radiologist. The number of ultrasounds referred to radiologists and adverse events within 1 week were recorded. The complication rate was compared with the published literature.
\end{abstract}

Results 960 ultrasound scans occurred over a 3 year period. The activity of the service increased over time, as a result of increased use of interventional ultrasound. The referral rate to radiology remained constant over the study period (mean proportion 4.0\%). Physician-delivered ultrasound correctly identified the presence/absence of pleural fluid in 951 of 955 evaluable scans $(99.6 \% \mathrm{Cl}$ $98.9 \%$ to $99.9 \%)$. The major complication rate was $3 /$ $558=0.5 \%$ (95\% Cl $0.1 \%$ to $1.6 \%)$, which compared favourably with the identified published literature. Conclusion Respiratory physician-delivered thoracic ultrasound appears to be safe and effective in the diagnosis/intervention of pleural effusion, and is associated with a major complication rate comparable with that of published studies. Continued liaison with the radiology service has here been demonstrated as a requirement for a physician-based service.

\section{INTRODUCTION}

Pleural effusions are a common problem in both general and respiratory medicine, with pleural fluid aspiration and chest tube insertion being among the most frequently performed medical procedures. The first diagnostic step for an effusion is aspiration for cytological, biochemical and microbiological analysis. ${ }^{1}$ Thoracic ultrasound has been shown to be superior to both chest radiography ${ }^{2}$ and clinical examination $^{3}$ in the detection of pleural fluid, and ultrasound-guided pleural aspiration and drain insertion have high success and low adverse event rates. ${ }^{4} 5$ A recent study in the USA has demonstrated lower pleural procedure complication rates with the use of thoracic ultrasound by respiratory physicians. $^{6}$ In the UK, thoracic ultrasound has recently been advocated in all cases to minimise adverse events. ${ }^{7}$ One strategy to achieve this effectively would be ultrasound performed by respiratory physicians at the bedside; however, this practice is not widespread in the UK and carries a potential risk where the operator has a narrower skill set than a more broadly trained radiologist. There have been no studies prospectively assessing safety or diagnostic accuracy for the detection of pleural fluid in UK operators trained to national standards. ${ }^{8}$

Our unit has run a respiratory physician-delivered ultrasound service since January 2006 using operators trained by thoracic-specific radiologists according to UK Royal College of Radiology (RCR) standards $^{8}$ for physician-based ultrasound. This prospective study assesses the safety and diagnostic accuracy for pleural fluid detection of this service, specifically adressing:

- The numbers of diagnostic and interventional respiratory physician-delivered ultrasound procedures performed over time

- The diagnostic accuracy of respiratory physician-delivered ultrasound for the detection of pleural fluid compared with a reference standard (see Methods section).

- The frequency with which radiological advice was required to manage complex cases.

- Adverse event rates and comparison with published studies derived from a review of the literature (see online supplement).

\section{METHODS}

The study was conducted at the Pleural Diseases Unit, Oxford, UK, which is a tertiary referral centre for pleural disease. The centre receives referrals from the local population (including from general medicine and oncology services) and regionally for pleural diagnostic and therapeutic procedures including tube thoracostomy, indwelling pleural catheter insertion, image-guided pleural biopsy and medical thoracoscopy. The ultrasound examinations were conducted by three respiratory physician operators trained to level I RCR guideline standards by two thoracic-specific radiologists (FVG and RB) (NMR from January 2006, HED from June 2007, JMW from March 2008). All patients requiring diagnostic ultrasound for pleural effusion or any interventional pleural procedure are included in this study between January 2006 and December 2008. Assessment of known pneumothorax was not included in this study. 
All ultrasound examinations were conducted on a Siemens Sonoline G60 machine, using a 2-6 Hz curvilinear abdominal probe. Patients were scanned in the upright position.

\section{Study protocol}

The following details were recorded for all examinations:

- Indication for thoracic ultrasound (prethoracoscopy, intervention (aspiration or drain insertion) or diagnostic (ie, referred for assessment of the presence of pleural fluid)).

- Operator.

- Findings (effusion present, size (small, effusion not seen $>2$ rib spaces above the posterior hemidiaphragm; moderate, effusion seen posteriorly to mid-thorax; large, effusion seen posteriorly above mid-thorax on ultrasound)).

- Interventions if performed.

- The need for thoracic radiology input (for diagnostic or procedural assistance) as judged by the thoracic ultrasound operator. Thoracic radiology input was requested for diagnostic scans where the operator was not confident of the sonographic appearances, and for interventional scans where the respiratory physician operator was not able to obtain pleural fluid despite supportive sonographic appearances.

- Major complications (defined as iatrogenic pneumothorax requiring chest drain insertion or aspiration, perforation of a viscous or clinically significant haemorrhage resulting in pain requiring analgesia if extrapleural, or signs of tachycardia or hypotension potentially requiring intervention).

- Pleural infection which developed within 1 week of any procedure (diagnosed as a clinically compatible history in association with acidic ( $\mathrm{pH}<7.2)$, purulent or microbiologypositive pleural fluid)

\section{Assessment of diagnostic accuracy}

Diagnostic accuracy of the ultrasound examination for the identification of pleural fluid was defined in a stepwise, pragmatic approach by the following criteria:

1. Pleural fluid successfully obtained at the time of the ultrasound.

2. Pleural fluid identified at thoracic ultrasound and successfully obtained at a subsequent procedure within 7 days (where no procedure was conducted during the initial ultrasound, due to clotting abnormality or convenience for the patient).

3. Confirmation of ultrasound findings by additional imaging modalities, reported by a radiologist (as part of routine clinical care), prior to and within 7 days of the ultrasound).

4. Chest radiographs reported by a thoracic radiologist showing consistent evidence of pleural effusion, blind to the ultrasound result and conducted prior to the thoracic ultrasound examination.

5. In the absence of any of the above, anonymised video clips/ still images of respiratory physician-delivered ultrasounds were reviewed by a consultant radiologist (RB), blind to clinical details and the ultrasound examination report. The radiologist assessment of pleural fluid was compared with the respiratory physician-delivered ultrasound report.

In a nested substudy, the above anonymised ultrasound images were separately scored blind to the respiratory physician ultrasound report by a physician operator (NMR) and radiologist (RB). Assessment was made of operator agreement, in terms of whether aspiration of fluid was considered technically possible assuming a clinical need to aspirate fluid.

\section{Literature review to define published adverse event rates}

A Medline search was conducted searching for all studies in which thoracic ultrasound was used for procedure guidance for pleural effusion. Studies of lung biopsy, pleural biopsy and pneumothorax were excluded. Those studies reporting complication rates only were included in the summary. For full details of the search strategy, studies included and excluded and the rationale behind this, see the online supplement.

\section{Statistical analysis}

SPSS version 14.0 was used for all statistical analysis. $\chi^{2}$ Analysis for proportions, linear regression, $\kappa$ values and CIs were used as appropriate to the data. For further details of statistical analysis, please see the online supplement.

\section{Ethics approval}

The study was approved by the Chairman of the Oxford Ethics Committee as an audit of clinical practice, with no requirement for informed consent.

\section{RESULTS}

Ultilisation of respiratory physician-delivered ultrasound service Over the 3 year study period, a total of 960 scans were performed in 645 patients (mean age 64 years, SD 17, 44\% female).

Respiratory physician-based ultrasound was conducted for the following indications: $246 / 960$ (25.6\%) diagnostic imaging only, $156 / 960$ (16.3\%) prethoracoscopy and 558/960 (58.1\%) interventional (aspiration in 244/960 (25.4\%); intercostal drain insertion ocurred in 314/960 (32.7\%))

The size of the effusion was recorded in 883/960 examinations $(92.0 \%)$; where not recorded, this was due to incomplete filling in of the ultrasound report by the respiratory physician operator. In $17(1.9 \%)$ there was no effusion, in 254 (28.8\%) the effusion was small, in 366 (41.4\%) the effusion was moderate and in 240 $(27.2 \%)$ the effusion was large. In three cases, a hydropneumothorax was diagnosed, and in three further cases a pneumothorax (unsuspected) alone was diagnosed (usually as a result of pleural drainage in a patient with a trapped lung). Of all interventional ultrasounds in which size was recorded (not including thoracoscopy, $\mathrm{n}=537), 131(24.4 \%)$ were conducted in small effusions and 406 (75.6\%) were conducted in moderate or large effusions.

The number of examinations conducted significantly increased over time from 25 scans in the first 3 months to 129 scans in the last 3 months (linear regression of 3 month period by number of ultrasounds; increase of 9.2 scans per 3 month period, $\mathrm{p}<0.001$ ) (figure 1). The number of examinations performed prethoracoscopy (mean number per month $=4.3, \mathrm{SD}$ 2.2) and for diagnostic purposes (mean number per month $=6.8$, SD 5.9) did not significantly increase over time (linear regression of 3 month period by number of ultrasounds, $p=0.77)$. In contrast the number conducted for intervention (aspiration or drain insertion) increased over time (figure 1) (linear regression of 3 month period by number of interventional ultrasounds; increase of 9.2 scans per 3 month period, $\mathrm{p}<0.001$ ).

\section{Radiology referral rate}

The frequency with which radiologist assistance was needed for the respiratory physician-based examination was measured as a proportion of all diagnostic and interventional (drain insertion/aspiration) ultrasounds. Scans conducted 


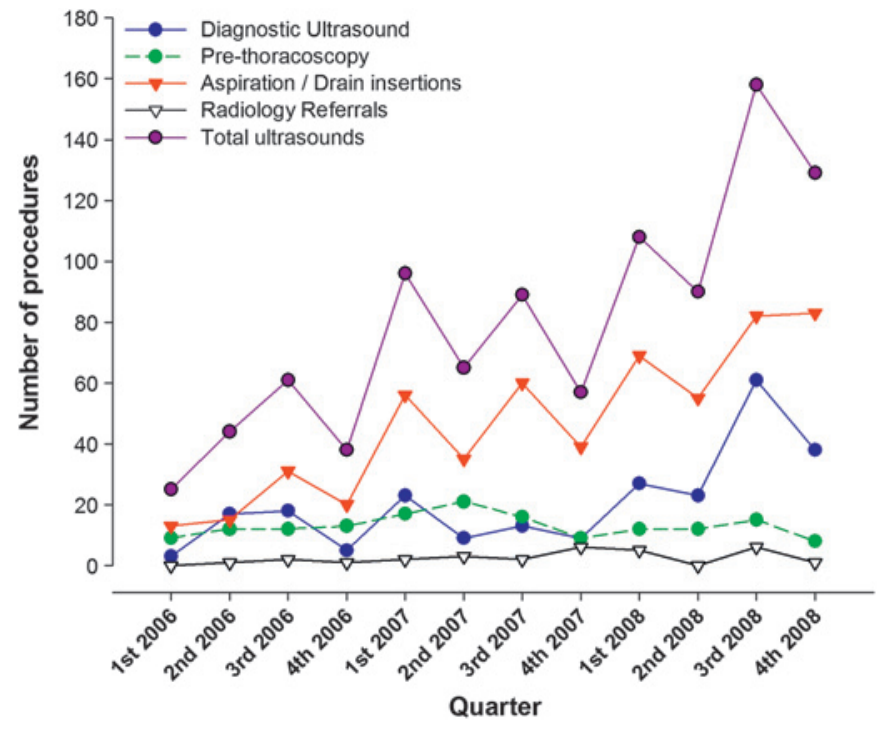

Figure 1 Increased activity of physician-delivered thoracic ultrasound over time, showing consistent radiology referral rates.

prethoracoscopy $(n=156)$ and preindwelling catheter insertions $(\mathrm{n}=83)$ were excluded from this analysis (as radiologists would not normally be involved in these procedures).

Radiological assistance was required in 29/721 (4.0\%, 95\% CI $2.7 \%$ to $5.7 \%$ ) cases, including $17 / 475(3.6 \%, 95 \% \mathrm{CI} \% 2.1$ to $5.7 \%$ ) for intervention and $12 / 246(4.9 \%, 95 \%$ CI $2.5 \%$ to $8.4 \%)$ for diagnostic interpretation. The proportion of ultrasounds referred to radiology remained constant over time (mean proportion $=3.4 \%$, SD 4.6, linear regression of month by proportion of referred ultrasound $\mathrm{p}=0.08$ ) (figure 1 ).

There was no significant difference in the proportion of ultrasound examinations referred to radiology in terms of size of effusion for interventional (referral proportion: 6/103 (5.5\%) small effusions, $9 / 339(2.6 \%)$ moderate or large effusions, $\chi^{2}, 1$ $\mathrm{df}=2.42, \mathrm{p}=0.12)$ or diagnostic (referral proportion $4 / 95(4.0 \%)$ small effusions, $3 / 101(2.9 \%)$ moderate or large effusions, $\chi^{2} 1$ $\mathrm{df}=0.22, \mathrm{p}=0.64)$ examinations. There was no difference in the radiology referral rate according to respiratory physician operator (data not shown).

\section{Diagnostic accuracy}

\section{Identification of pleural fluid}

Data regarding diagnostic accuracy of fluid identification by respiratory physician ultrasound was available in 955/960 $(99.5 \%)$ of scans, with efficacy data missing in five cases (in these five cases, clips were not recorded due to machine failure) (figure 2). Combining all criteria for diagnostic accuracy of respiratory physician ultrasound to detect pleural fluid (fluid aspirated, radiological imaging, radiologist review of clips), respiratory physician-delivered ultrasound was correct in diagnosing the presence or lack of pleural fluid in 951/955 $(99.6 \%$, $95 \%$ CI $98.9 \%$ to $99.9 \%$ ) of evaluable cases (figure 2 ).

\section{Details of radiologist review of ultrasound images/clips}

A total of 47 scans in which no other diagnostic accuracy confirmation was available were reviewed by a radiologist. For the detection of pleural fluid, blind review by a radiologist was in agreement with the respiratory physician ultrasound report in 43/47 (91.5\%) cases (table 1), with 33 true positives, 10 true negatives and four false negatives (ie, physician ultrasound failed to detect pleural fluid in four cases where the radiologist detected fluid). This provides an overall sensitivity for detection of pleural fluid in this subset of $33 / 37(89.2 \%)$ and a specificity of $10 / 10(100 \%)$. There were no false positives (ie, detection of pleural fluid at physician ultrasound not confirmed by the radiologist).

Assessing technical feasibility of pleural fluid for aspiration, the radiologist review was in agreement with the respiratory physician operator in 42/47 (89.3\%) cases (table 2). There were five false negatives (ie, radiologist reported effusion technically possible to aspirate, physician did not) and no false positives (ie, physician suggested aspiration technically possible where radiologist did not). The overall sensitivity of respiratory physician opinion for detecting effusion feasibility for aspiration was 29/ $34(85.3 \%)$ with a specificity of $13 / 13(100 \%)$. In one of the falsenegative cases, the effusion was not recognised by the respiratory physician (heavily consolidated lung adjacent to small effusion), and in the other four cases the effusion was considered by the respiratory physician to be too small to aspirate (mean depth of effusion in these five cases $=1.1 \mathrm{~cm}, \mathrm{SD} 0.6 \mathrm{~cm}$ ).

The $\kappa$ value comparing radiologist and respiratory physician for effusion detection was 0.78 (95\% CI 0.57 to $0.97, \mathrm{p}<0.001)$ and for amenable to aspiration it was 0.76 (95\% CI 0.57 to 0.96 , $\mathrm{p}<0.001)$.

\section{Complications}

The overall major complication rate was 3/558 (0.5\%, 95\% CI $0.1 \%$ to $1.6 \%)$. All complications occurred in the ultrasound intervention group $(n=558)$. These were two cases of intrapleural bleeding requiring intervention, and one case of pleural infection (with no deaths directly attributable to the procedures). There were no instances of pneumothorax requiring intervention or lung or visceral perforation.

\section{Literature review of ultrasound-guided complications}

For details of the literature review of ultrasound-guided pleural procedure complications, please see the online supplement.

\section{Discussion}

This is the first large systematic prospective description of the diagnostic accuracy and safety of a respiratory physician-delivered thoracic ultrasound service. The results show a high diagnostic accuracy for the identification of pleural fluid Comparison with the published literature of complications from ultrasound-guided pleural procedures shows an adverse event rate in this study at least as low as that seen in large published series of ultrasound-based intervention for pleural effusions (figure 3 and online supplement). The overall major complication rate reported in the identified studies was 137 complications in 6836 procedures $(2.0 \%, 95 \%$ CI $1.7 \%$ to $2.3 \%)$. The major complication rate of radiology studies $(121 / 5530=2.2 \%, 95 \%$ CI $1.8 \%$ to $2.6 \%$ ) was comparable with that of the physicianconducted studies $(16 / 1306=1.2 \%, 95 \%$ CI $0.7 \%$ to $2.0 \%)\left(\chi^{2} 1\right.$ $\mathrm{df}=5.0, \mathrm{p}=0.03)$. The proportion of complications seen in this study was significantly lower than the proportion in published studies (this study $3 / 558,0.5 \%$ vs $137 / 6836,2.0 \% ; \chi^{2} 1 \mathrm{df}=6.0$, $\mathrm{p}=0.01$, difference $1.5 \%, 95 \%$ CI for the difference $0.8 \%$ to $2.2 \%$ ) (figure 3).

'Point of care' ultrasound allows immediate patient access to the potential advantages of improved safety, diagnostic accuracy and reduced treatment delays. For the examinations to be performed by physicians would also add to this flexibility, but this would result in examinations being performed by operators 
Figure 2 Flowchart of diagnostic accuracy assessment methods used for physician-delivered ultrasound, showing a high overall accuracy for the detection of pleural fluid.

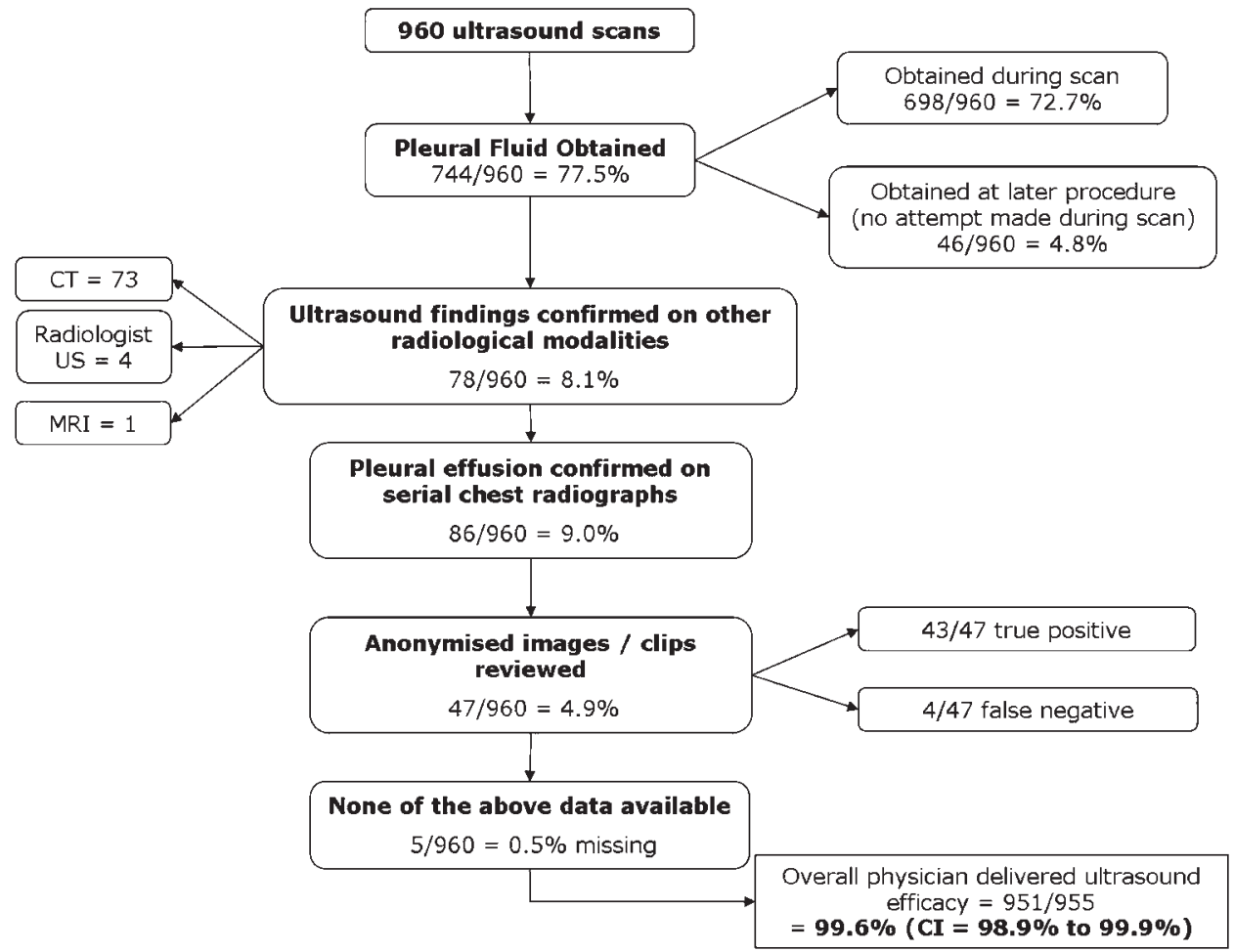

without the broad training of radiologists. Therefore, large studies of physician-delivered 'point of care' ultrasound are required before this approach is widely used. The results of this study are encouraging in this regard.

The activity of respiratory physician-delivered ultrasound increased over time in this study, due to increased interventional (drain insertion and aspiration) activity. This was due to the service becoming better utilised by allied, non-respiratory specialities and an increased awareness of complications.

Ultrasound has been demonstrated to be a safer and more effective technique than clinical examination alone for the detection of pleural fluid and for guiding interventions, ${ }^{4-6}$ and its use by chest physicians is likely to increase. The recent UK National Patient Safety Agency report on complications from chest drain insertion ${ }^{7}$ highlights the potential hazards from this common procedure. In light of this report, it is likely that there will be a move towards the majority of pleural interventions occurring under ultrasound guidance, and the pattern of increase seen in this study reflects this.

The referral rate to radiology colleagues from the physicianbased ultrasound service $(4.0 \%)$ remained constant over time. It may be assumed that as physician ultrasound operators become more familiar with the technique, referral rates would decrease, but we have not found this to be the case. It is likely that more complicated cases were undertaken by the physician service over time, maintaining the constant radiology referral rate, although this aspect has not been specifically addressed in this study. These results suggest that continued support from an expert

Table 1 Comparison of physician with blind radiologist review in the detection of pleural fluid in the 47 scans reviewed

\begin{tabular}{lllll}
\hline & & Radiologist score & \\
\cline { 3 - 4 } & & Effusion present & Effusion absent & Totals \\
\hline Physician score & Effusion present & 33 & 0 & 33 \\
& Effusion absent & 4 & 10 & 14 \\
& Totals & 37 & 10 & $\mathbf{4 7}$ \\
\hline
\end{tabular}

radiology service is essential for a physician-delivered service to function safely and effectively, even in a service with a high number of diagnostic and interventional ultrasound procedures. Accurate assessment of radiology referral rate in a non-specialist centre is required to provide generalisable results.

We have measured diagnostic accuracy of ultrasound using a stepwise pragmatic approach. All ultrasounds were conducted for the assessment of pleural effusion, and therefore detection of this feature was specifically targeted for this study. The overall diagnostic accuracy for the detection of pleural fluid by physicians using ultrasound reported here is high (99.6\%). Information on diagnostic accuracy was missing in five cases; however, even adopting a conservative approach and including these missing cases as physician ultrasound 'failures', the overall diagnostic accuracy remains high $(951 / 960=99.1 \%$, 95\% CI $98.2 \%$ to $99.6 \%$ )

The results of the subset of examinations which directly compared radiologist and respiratory physician interpretation of ultrasound clips/images $(n=47)$ is reassuring. Although physician ultrasound failed to recognise the presence of pleural fluid in 4/37 cases, these effusions were small and adjacent to consolidated lung. We are not able here to address specifically whether this small number of false-negative ultrasound scans would have resulted in adverse patient outcomes.

There were no 'false positives', suggesting safe practice. Direct comparison of whether fluid was amenable to aspiration suggests a more conservative approach by physician operators

Table 2 Comparison of radiologist score with physician score for whether effusions were amenable to aspiration in 47 cases reviewed

\begin{tabular}{llrcr}
\hline & Radiologist score & & & \\
\cline { 2 - 4 } & & $\begin{array}{l}\text { Aspiration } \\
\text { possible }\end{array}$ & $\begin{array}{l}\text { Aspiration } \\
\text { not possible }\end{array}$ & Totals \\
\hline Physician score & Aspiration possible & 29 & 0 & 29 \\
& Aspiration not possible & 5 & 13 & 18 \\
& Totals & 34 & 13 & 47 \\
\hline
\end{tabular}




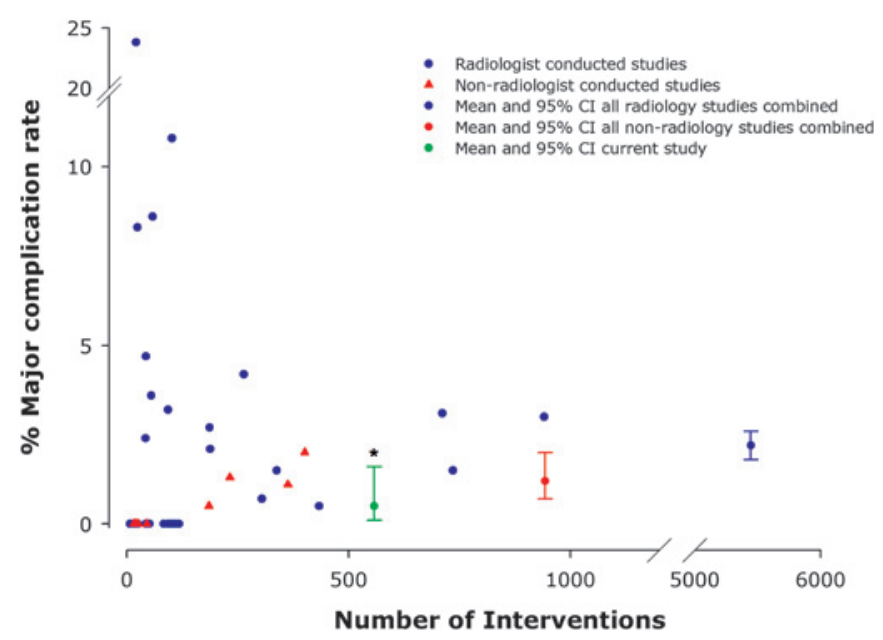

Figure 3 Major complication rates of studies using ultrasound for pleural fluid procedures, assessing studies by size and whether conducted by a radiologist or non-radiologist, in comparison with the current study. Individual studies are shown with filled triangles, and summary complication rates (non-weighted) and $95 \% \mathrm{Cl}$ are shown with dots and bars (see key). For detailed literature search results, see the online supplement.

compared with experienced radiologists-there were no instances of physician operators reporting an effusion as amenable to aspiration where radiologists did not. We believe it is appropriate that radiologists with greater experience and expertise in ultrasound have a lower threshold for intervention. In this study, 5/34 cases were considered too small for aspiration by physician operators where radiologists considered these to be amenable to aspiration, and all of these effusions were small (mean depth of fluid $1.1 \mathrm{~cm}$ ).

Physician-based thoracic ultrasound is increasing in the USA, Europe and the UK. There are published guidelines on training on these continents, ${ }^{9} 10$ but there are few published data to confirm that they result in safe practice, and there are reasonable concerns that a short period of training in ultrasound may be insufficient. The results of this study suggest that the major complication rate of level I UK RCR-trained physicians is comparable with previously published studies of both physicianand radiology-based ultrasound-guided pleural procedures in this cohort of patients. Although the reported complication rate from this study $(0.4 \%)$ appears lower than the published standard, it is possible that less complicated cases were taken on in this study, especially compared with the radiology literature. In addition, all procedures were here performed by three operators-it is possible that the low complication rate here reflects interventions conducted by a small number of trained operators as has been seen in previous studies, ${ }^{6}$ and our data do not allow this to be specifically assessed.

The increasing use of thoracic ultrasound by respiratory physicians may result in the majority of such scans being performed by physicians rather than radiologists. This raises the potential issue of reduced experience in thoracic ultrasound for radiology trainees in those centres where the majority of the workload is undertaken by physicians. In such centres, strategies such as joint physician/radiologist training may be required to maintain thoracic ultrasound skills within both services, with training provided by both radiologists and respiratory physicians with substantial experience ${ }^{8}$ in thoracic ultrasound.
There are several limitations to this study. The study was conducted in a tertiary referral centre for pleural disease, with a high workload of pleural effusions and intervention. In nonspecialist centres, the number of ultrasounds conducted may be significantly lower, which may decrease the number of scans conducted. In addition, all physician ultrasound scans in this study were conducted by three operators over a 3 year period, whereas most hospitals will have a higher throughput of staff who individually undergo shorter periods of training and experience. Support and training in drain insertion technique, complication management and continued liaison and referral to radiologists when required are important aspects to be considered.

There is no published, accepted standard for the complication rate of ultrasound-guided pleural procedures, and we have reviewed the literature in an attempt to address this question. It should be accepted that this was not a formal systematic review and no formal statistical techniques were used to attempt data synthesis; it is possible that studies addressing this question were not identified. Taking this into account, the complication rate identified in this study was reassuring and comparable with the identified literature. Further formal assessment of studies addressing complication rates from ultrasound-guided procedures is now required.

\section{Conclusions}

Physician-based ultrasound appears to be safe and effective for diagnosis and intervention in patients with pleural effusion. The major complication rate appears to be comparable with published studies of physician- and radiologist-operated pleural ultrasound. The requirement for continued close liaison with the radiology service has here been demonstrated as a requirement for a physician-based service.

Funding NMR is funded by the UK Medical Research Council, EKM is funded by the UK National Cancer Research Institute and RJOD is funded by the NIHR Oxford Biomedical Research Centre programme.

\section{Conflict of interest None.}

Ethics approval The study was approved by the Chairman of the Oxford Ethics Committee as an audit of clinical practice, with no requirement for informed consent.

Provenance and peer review Not commissioned; externally peer reviewed.

\section{REFERENCES}

1. Maskell NA, Butland RJ. BTS guidelines for the investigation of a unilateral pleura effusion in adults. Thorax 2003;58 (Suppl 2):ii8-17.

2. Eibenberger KL, Dock WI, Ammann ME, et al. Quantification of pleural effusions: sonography versus radiography. Radiology 1994;191:681-4.

3. Diacon $\mathbf{A H}$, Brutsche $\mathbf{M H}$, Soler M. Accuracy of pleural puncture sites: a prospective comparison of clinical examination with ultrasound. Chest 2003; 123:436-41.

4. O'Moore PV, Mueller PR, Simeone JF, et al. Sonographic guidance in diagnostic and therapeutic interventions in the pleural space. AJR Am J Roentgenol 1987; 149:1-5

5. Jones PW, Moyers JP, Rogers JT, et al. Ultrasound-guided thoracentesis: is it a safer method? Chest 2003;123:418-23.

6. Duncan DR, Morgenthaler TI, Ryu JH, et al. Reducing iatrogenic risk in thoracentesis: establishing best practice via experiential training in a zero-risk environment. Chest 2009:135:1315-20.

7. Anon. Risk of chest drain insertion. UK: National Patient Safety Agency, 2009

8. Royal College of Radiology. Ultrasound training recommedations for medical and surgical specialties. UK: Royal College of Radiology, 2005.

9. American College of Radiology. ACR practice guideline for performing and interpreting diagnostic ultrasound examinations. American College of Radiology, 2006 http://www.acr.org/SecondaryMainMenuCategories/quality_safety/guidelines/us/ us_performing_interpreting.aspx

10. European Federation of Societies for Ultrasound in Medicine and Biology Minimum training requirements for the practise of medical ultrasound in Europe. Appendix 11: thoracic ultrasound, 2009. http://www.efsumb.org/guidelines/ guidelines01.asp 